Pacific Journal of Mathematics

CONGRUENCE LATTICES OF ALGEBRAS OF FIXED Ample and Walter Fuller Taylor 


\title{
CONGRUENCE LATTICES OF ALGEBRAS OF FIXED SIMILARITY TYPE, I
}

\author{
Ralph Freese, William A. Lampe and Walter Taylor
}

\begin{abstract}
We prove that if $V$ is any infinite-dimensional vector space over any uncountable field $F$, then the congruence lattice (=subspace lattice) of $V$ cannot be represented as a congruence lattice (of any algebra) without using at least $|F|$ operations. This refutes a long-standing conjecture-that one binary operation would always suffice.
\end{abstract}

Our result implies that the natural representation of Sub $V$ as a congruence lattice is the best one, i.e., uses the minimum number of operations. This result is easy to obtain-see $\S 1$.

In the remainder of the paper we find further necessary conditions for the representability of an algebraic lattice $L$ with $<\kappa$ operations. Necessary and sufficient conditions seem impossible at this stage of knowledge. Part II of this paper (by W. A. Lampe) gives some interesting sufficient conditions for representability of $L$ with one binary operation, for instance: the unit element of $L$ is compact.

The conjecture which we have refuted dates back at least to 1959 and the theorem of Grätzer and Schmidt ([6], [3], [14]) that every algebraic lattice can be represented as the congruence lattice Con $\boldsymbol{A}$ of some unary algebra $A$; the hope was to use say a single binary operation + to code the unary operations $f(x)$ as $x+a$ (with $a \in A$ depending on $f$ ). (See e.g., [9], [8, p. 209].)

By some results of $\S 3$ and Part II, every algebraic lattice can be embedded as a principal ideal in an algebraic lattice which is representable with one binary operation, and also in one requiring $\kappa$ operations. This may partly explain why the conjecture resisted settlement for so long.

$\S 1$ contains the main result. $\S \S 2$ and 3 contain refinements and variations on the ideas of $\S 1$, and $\S 4$ contains some open problems.

Some of these results were announced in [12], [13], and [18].

The authors thank B. Jónsson, A. Day, and E. Nelson for many helpful comments, and acknowledge support from the National Science Foundation and support (for W. A. Lampe) from the Institute for Advanced Study during 1974-75. The other two authors heartily thank William Lampe for so successfully initiating this investigation, and especially for his crucial Lemma 1, which made everything here possible. 
1. The main result. All our results depend on the following lattice-theoretic property:

(*) For every compact $\Psi$, there exist compact $\Theta, \Phi$ such that $\Theta \vee \Phi \geqq \Psi$ and $\Theta \wedge \Psi=\Phi \wedge \Psi=0$.

It is easy to see that our subspace lattice obeys $(*)$ (see the proof of Theorem 1); in fact all examples in this article will obey (*).

Lemma 1. If Con $\boldsymbol{A}$ satisfies (*), then $\boldsymbol{A}$ satisfies

$$
\exists x(t(x, p)=t(x, q)) \longrightarrow \forall x(t(x, p)=t(x, q))
$$

(for $x$ denoting $\left(x_{1}, \cdots, x_{n}\right), p, q \in A^{m}$ and $t$ any $(n+m)$-ary term, i.e., polynomial).

REMARKs. (*) is somewhat elusive-every algebraic lattice can be embedded as a principal ideal in an algebraic lattice obeying (*), and also in one where $(*)$ fails. And so perhaps it is not surprising that Lemma 1 was overlooked for so long. It arose from a study of the lattices $L_{\lambda, \phi}$ of $\S 2$.

Proof. We will assume that $t(a, p)=t(a, q)$ for some $a \in A^{n}$, and prove that $t(b, p)=t(b, q)$ for any $b \in A^{n}$. To see this, we will apply (*) to the compact congruence $\Psi=\Theta(a, b) \vee \Theta(p, q)$ (i.e., the congruence generated by $\left.\left\{\left(a_{1}, b_{1}\right), \cdots,\left(a_{n}, b_{n}\right),\left(p_{1}, q_{1}\right), \cdots,\left(p_{m}, q_{m}\right)\right\}\right)$. Taking $\Phi$ and $\theta$ as supplied by $(*)$, we have

$$
\Theta(a, b) \subseteq \Psi \subseteq \Theta \vee \Phi,
$$

and so

$$
a=s_{0} \Theta s_{1} \Phi s_{2} \Theta s_{3} \Phi \cdots s_{k}=b .
$$

Now since $s_{0} \Theta s_{1}$, we have

$$
t\left(s_{1}, p\right) \Theta t\left(s_{0}, p\right)=t\left(s_{0}, q\right) \Theta t\left(s_{1}, q\right),
$$

and thus $t\left(s_{1}, p\right) \Theta t\left(s_{1}, q\right)$. Since $p \Psi q$, we also have $t\left(s_{1}, p\right) \Psi t\left(s_{1}, q\right)$. Finally, since $\Theta \cap \Psi=0$, we have $t\left(s_{1}, p\right)=t\left(s_{1}, q\right)$. A similar argument (with $\Phi$ in place of $\Theta$ ) yields $t\left(s_{2}, p\right)=t\left(s_{2}, q\right)$. Proceeding by induction, we obtain $t(b, p)=t\left(s_{k}, p\right)=t\left(s_{k}, q\right)=t(b, q)$. of $\boldsymbol{A}$.

Definition. $\# \boldsymbol{A}$ denotes the number of nonnullary operations

Lemma 2. If Con $\boldsymbol{A}$ obeys (*), then every block of every compact congruence of $\boldsymbol{A}$ has power $\leqq \boldsymbol{A}+\boldsymbol{\aleph}_{0}$. 
Proof. Let $S$ be a block of a compact congruence $\Theta$; thus $\Theta=$ $\Theta(a, b)$ for some $a, b \in A^{n}$. Fixing $c \in S$ and considering arbitrary $d \in S$, we have $(c, d) \in \Theta(a, b)$, and hence, for some terms $t_{1}, \cdots, t_{n}$ and $p_{1}, \cdots, p_{n} \in A^{m}$, we have

$$
\begin{aligned}
c & =t_{1}\left(a, b, p_{1}\right) \\
t_{1}\left(b, a, p_{1}\right) & =t_{2}\left(a, b, p_{2}\right) \\
& \vdots \\
t_{n-1}\left(b, a, p_{n-1}\right) & =t_{n}\left(a, b, p_{n}\right)=d
\end{aligned}
$$

(as follows from well known and easy descriptions of compact congruences-see e.g., [3, Theorem 10.4]).

To see our upper bound on $|S|$, it is clearly enough (by elementary cardinal arithmetic) to see that $d$ depends only on the sequence $\left(t_{1}, \cdots, t_{n}\right)$, and not on $\left(p_{1}, \cdots, p_{n}\right)$. To see this we suppose we also have

$$
\begin{aligned}
c & =t_{1}\left(a, b, p_{1}^{\prime}\right) \\
t_{1}\left(b, a, p_{1}^{\prime}\right) & =t_{2}\left(a, b, p_{2}^{\prime}\right) \\
& \vdots \\
t_{n-1}\left(b, a, p_{n-1}^{\prime}\right) & =t_{n}\left(a, b, p_{n}^{\prime}\right)=d^{\prime} .
\end{aligned}
$$

The proof will be complete when we have shown $d=d^{\prime}$. From $t_{1}\left(a, b, p_{1}\right)=c=t_{1}\left(a, b, p_{1}^{\prime}\right)$ we obtain

$$
t_{2}\left(a, b, p_{2}\right)=t_{1}\left(b, a, p_{1}\right)=t_{1}\left(b, a, p_{1}^{\prime}\right)=t_{2}\left(a, b, p_{2}^{\prime}\right),
$$

(using Lemma 1 for the middle equality). Thus we have obtained $t_{2}\left(a, b, p_{2}\right)=t_{2}\left(a, b, p_{2}^{\prime}\right)$; proceeding by induction yields $d=d^{\prime}$.

The above lemma tells us that blocks cannot be too big. The next lemma says that sometimes there must be a large block.

Lemma 3. Suppose that $\Psi \in \operatorname{Con} \boldsymbol{A}, X \subseteq \operatorname{Con} \boldsymbol{A}$ is infinite, and for distinct $\Theta, \Phi \in X, \Theta \wedge \Phi=0$ and $\Theta \vee \Phi=\Psi$. Then each nontrivial block of $\Psi$ has power $\geqq|X|$.

Proof. Let $I$ be any partition of $X$ into two-element sets, and take $\langle c, e\rangle \in \Psi$ with $c \neq e$. For each $B \in \Pi, \vee B=\Psi$, and so we have

$$
c \Theta_{1} d \Theta_{2} \cdots \Theta_{k} e \quad\left(\text { each } \theta_{i} \in B\right) .
$$

Of course we may assume that $d \neq c$. It will be enough to see that distinct $B$ 's yield distinct $d$ 's (for then the set of all $d$ 's has power $\geqq|\Pi|=|X|$, and certainly $c \Psi d$ holds for each $d)$. But this is easy, since if we also had $c \Theta_{1}^{\prime} d$ with $\Theta_{1}^{\prime} \in B^{\prime} \neq B$, then we would have $c=d$, since $\Theta_{1} \cap \Theta_{1}^{\prime}=0$. 
THEOREM 1. If $V$ is an infinite-dimensional vector space over an uncountable field $\boldsymbol{F}$, then $\operatorname{Con} \boldsymbol{A} \cong \operatorname{Con} V$ implies that $\boldsymbol{A}$ has at least $|F|$ operations.

Proof. We will see that $\operatorname{Con} V$ (hence $\operatorname{Con} \boldsymbol{A}$ ) satisfies the hypotheses of Lemma 3, with $\Psi$ actually compact. Fix one 2-dimensional subspace $W$ and $|F|$ one-dimensional subspaces $U_{\alpha} \subseteq W(\alpha<|F|)$. With the usual identification of subspaces with congruences, we take $\Psi=W$ and $X=\left\{U_{\alpha}: \alpha<|F|\right\}$. Clearly $U_{\alpha} \vee U_{\beta}=W$ for $\alpha \neq \beta$, and so Lemma 3 yields a compact congruence with a block of power $|F|$.

To see that $\operatorname{Con} V$ (=Sub $V$ ) obeys (*), let $U$ be any finite-dimensional subspace of $V$ with basis $\left\{v_{1}, \cdots, v_{n}\right\}$. Extend this to a linearly independent subset $\left\{v_{1}, \cdots, v_{2 n}\right\}$ of $V$. Now take $U^{\prime}$ to be spanned by $\left\{v_{1}+v_{n+1}, \cdots, v_{n}+v_{2 n}\right\}$ and $U^{\prime \prime}$ to be spanned by $\left\{v_{n+1}, \cdots, v_{2 n}\right\}$. Then certainly $U \subseteq U^{\prime}+U^{\prime \prime}$ and $U \cap U^{\prime}=U \cap U^{\prime \prime}=\{0\}$.

And so Lemma 2 tells us that $|F| \leqq \# A+\aleph_{0}=\# \boldsymbol{A}$.

2. Refinements. In this section we present further means of finding large congruence blocks and thus seeing (via Lemma 2) that certain algebraic lattices obeying (*) require many operations in their congruence representations. Lemma 4 below may be proved in a manner similar to Lemma 3 . Lemma 5 is far more general than Lemma 4, but also more complicated. Lemma 6 below produces large congruence blocks in an entirely different manner.

Lemma 4. Suppose that $\Psi \in \operatorname{Con} \boldsymbol{A}, X \subseteq \operatorname{Con} \boldsymbol{A}$ and a partition $\Pi$ of $X$ obey these conditions:

(1) for each $B \in \Pi, \Psi \leqq \vee B$;

(2) for distinct $B, B^{\prime} \in \Pi, \Theta \in B$ and $\Phi \in B^{\prime}, \Theta \wedge \Phi=0$.

Then every nontrivial block of $\vee X$ has power $\geqq|\Pi|$.

In what follows, $\kappa$ is an infinite cardinal. We say that a poset $P$ is $\kappa$-directed if and only if each $S \subseteq P$ with $|S|<\kappa$ has an upper bound in $P$.

Theorem 2. If Con $\boldsymbol{A}$ satisfies (*) and the compact elements of Con $\boldsymbol{A}$ are $\kappa^{+}$-directed, then $\kappa \leqq \# \boldsymbol{A}+\boldsymbol{\aleph}_{0}$.

Proof. By Lemma 2, it will be enough to produce a compact congruence with one block of power $\geqq \kappa$. For this, we use $(*)$ and $\kappa^{+}$-directedness. For $\alpha \leqq \kappa$ we recursively define compact congruences $\Psi_{\alpha}, \Theta_{\alpha}, \Phi_{\alpha}$ as follows: 


$$
\begin{aligned}
\Psi_{\alpha} & \geqq \Psi_{\beta}, \Theta_{\beta}, \Phi_{\beta} \quad(\alpha>\beta) \\
\Theta_{\alpha} \vee \Phi_{\alpha} & \geqq \Psi_{\alpha} \\
\Theta_{\alpha} \wedge \Psi_{\alpha} & =\Phi_{\alpha} \wedge \Psi_{\alpha}=0
\end{aligned}
$$

(the first condition is possible by $\kappa^{+}$-directedness, the last two by $(*)$ ). It is clear that if we take $X=\left\{\theta_{\alpha}, \Phi_{\alpha}: \alpha<\kappa\right\}$ and $\Pi=\left\{\left\{\Theta_{\alpha}, \Phi_{\alpha}\right\}: \alpha<\kappa\right\}$, then the hypotheses of Lemma 4 are fulfilled, and moreover $\vee X \subseteq \Psi_{k}$. Thus $\Psi_{\kappa}$ is a compact congruence with a block of power $\geqq \kappa$.

To illustrate Theorem 2, we suppose $V$ is a vector space of dimension $\kappa^{+}$over some field. We let $S$ be the lattice of all subspaces of $V$ of dimension $\leqq \kappa$, and let $L$ be the lattice of all ideals of $S$. Theorem 2 says that $\# \boldsymbol{A} \geqq \kappa$ whenever $L \cong \operatorname{Con} A$.

Lemma 5. Suppose that $\Psi, Z \in \operatorname{Con} \boldsymbol{A}$ and a family $P$ of finite subsets of Con $\boldsymbol{A}$ obey these conditions:

(1) $|\cup P|$ is regular and infinite;

(2) $\Psi$ is compact and $\Psi \geqq Z$;

(3) $\forall B \in P, \Psi \leqq(\vee B) \vee Z$;

(4) each $B \in P$ is minimal for condition (3);

(5) $\forall T \subseteq \cup P$, if $|T|=|\cup P|$, then $\wedge T \leqq Z$.

Then $\vee(\cup P) \vee Z$ has a block of power $\geqq|\cup P|$.

REMarks. Of course the finiteness of the sets $B \in P$ follows from (2) and (4). This lemma is more versatile than Lemma 4 since, firstly, $P$ need not be a partition, secondly, the disjointness condition (5) has been weakened considerably, and thirdly, 0 has been replace by an arbitrary $Z \geqq \Psi$. Obviously the new assumption (1) makes no difference for our applications in conjunction with Lemma 2, because each singular cardinal is the supremum of all smaller regular cardinals.

Proof. By (2) we have $a, b \in A^{n}$ with

$$
\Psi=\Theta(a, b)=\Theta\left(a_{1}, b_{1}\right) \vee \cdots \vee \Theta\left(a_{n}, b_{n}\right) .
$$

Since each $\left(a_{i}, b_{i}\right) \in \Psi$, (3) says that for each $B \in P$

$$
a_{i}=x_{0} \Theta_{1} x_{1} \Theta_{2} x_{2} \cdots x_{k}=b_{i}
$$

for some $x_{1}, x_{2}, \cdots \in A$ and some $\Theta_{1}, \Theta_{2}, \cdots \in B \cup\{Z\}$. For each $i$ we form $Q_{i} \subseteq A$ as follows: take one such sequence for each $B \in P$, and then let $Q_{i}$ be the set of all $x_{1}, x_{2}, \cdots$ occurring in all these sequences. Obviously each $Q_{i}$ is contained in a single $\vee(\cup P) \vee Z$ block, and so it will be enough to see that some $\left|Q_{i}\right| \geqq|\cup P|$.

Clearly it is enough to show that $Q=Q_{1} \cup \cdots \cup Q_{n}$ has power $\geqq|X|$, 
where $X=\cup P$. To see this we define

$$
\tau:(Q \times Q-Z) \longrightarrow \text { power set of } X
$$

via

$$
(p, q) \longmapsto\{\Theta \in X: p \Theta q\} \text {. }
$$

We will clearly be done if we can show that $\mid$ Range $\tau|\geqq| X \mid$. Condition (5) implies that each $|\tau(p, q)|<|X|$, and so by the regularity of $|X|$ it will be enough to see that $\mid \cup$ Range $\tau|=| X \mid$. We will in fact show that $\cup$ Range $\tau=X$.

For each $\Theta \in X=\cup P$, we have $B \in P$ and

$$
a_{i}=x_{0} \Theta_{1} x_{1} \Theta_{2} x_{2} \cdots x_{k}=b_{i} \quad(1 \leqq i \leqq n)
$$

(as above) with $\Theta \in B$ and each $\Theta_{j} \in B \cup\{Z\}$. Now among these relations must occur $x_{j} \Theta x_{j+1}$ with $\left(x_{j}, x_{j+1}\right) \notin Z$, for otherwise we could eliminate $\Theta$ in favor of $Z$, contradicting the minimality of $B$ in (4). But clearly $\Theta \in \tau\left(x_{j}, x_{j+1}\right)$, and hence $X \subseteq$ Range $\tau$.

Lemma 6. Suppose that $C$ is the set of compact elements of Con $\boldsymbol{A}, X \leqq$ Con $\boldsymbol{A}$ and $X$ is infinite. If $\vee X=1$ and $|X|<|C|$, then some congruence in $X$ has a block of power $\geqq \kappa$, for each regular $\kappa$ satisfying $|X|<\kappa \leqq|C|$. (In particular, for $\kappa=|X|^{+}$.)

Proof. Fixing such a regular $\kappa$, we let $Y$ be the set of finite joins of congruences from $X$. Thus $Y$ is directed and $|Y|<\kappa$. Fixing any $a \in A$, we have, since $A$ is the unique block of 1 ,

$$
A=[a]_{1}=\bigcup_{\theta \in Y}[a]_{\Theta} \text {. }
$$

One easily checks that $|C| \leqq|A|$, and so the regularity of $\kappa$ yields $\left|[a]_{\Theta}\right| \geqq \kappa$ for some $\Theta \in Y$.

By definition of $Y$, we have $\Theta=\Theta_{1} \vee \cdots \vee \Theta_{n}$ with each $\Theta_{j} \in X$. Let

$$
Q_{0}=\{a\} \text { and } Q_{i+1}=\left\{x \notin Q_{i}:\left(\exists y \in Q_{i}\right)(\exists j) x \Theta_{j} y\right\} \text {. }
$$

Since $[a]_{\Theta}=\bigcup_{i \in \omega} Q_{i}$ and $\kappa$ is regular, there exists $i$ with $\left|Q_{i+1}\right| \geqq \kappa$; taking the least such $i$, we also have $\left|Q_{i}\right|<\kappa$. For $b \in Q_{i}$ and each $j$, let $R_{b j}=\left\{x \in Q_{i+1}: b \Theta_{j} x\right\}$. By the regularity of $\kappa$, we may choose $b$ and $j$ with $\left|R_{b j}\right| \geqq \kappa$, and clearly $\left|[b]_{\Theta_{j}}\right| \geqq \kappa$.

To illustrate how Lemmate 5 and 6 may be applied, we will present several algebraic lattices, all of the same general form, namely: $L_{\alpha, K}$ is the ideal lattice of 


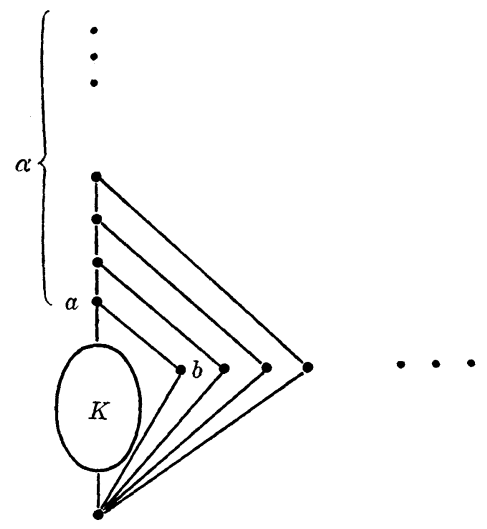

for a limit ordinal $\alpha$ and $a$ join-semilattice $K$ which we will specify further below. Obviously the lattices $L_{\alpha, K}$ satisfy (*), and so we may apply Lemma 2 to them.

We first note that if $\alpha=\beta+\gamma$ with $\gamma<|\beta|+|K|$, then Lemmate 2 and 6 immediately yield $\# A \geqq|\beta|+|K|$ for Con $A \cong L_{\alpha, K}$. An example where neither Lemma 4 nor Lemma 5 is useful is obtained by taking $\alpha=\omega$ and $K=\omega_{1}$.

By way of historical comment, we should remark that Lampe's pioneering example [12] was precisely $L_{\alpha, K}$ with $\alpha=\omega_{1}+\omega, K=\varnothing$. (He actually used Lemmate 2 and 4 , which apply equally well to this example.)

The lattices $L_{\alpha, \varnothing}$ arose quite naturally during Lampe's attempts to represent each algebraic lattice as the congruence lattice of a groupoid. It is possible to view $L_{\omega, \varnothing}$ as a partial lattice in such a way that a lattice $L$ obeys $(*)$ if and only if for every compact $a$ there is a zero-preserving embedding $\sigma$ of $L_{\omega, \varnothing}$ into $L$ with $a$ in the range of $\sigma$. Each of Lampe's attempts foundered on a lattice which had some $L_{\alpha, \varnothing}$ as a partial sublattice.

Next we give three examples using various features of Lemma 5, where neither Lemma 4 nor Lemma 6 can be applied. These examples have $\alpha=\omega_{1}$ and $|K|=\omega_{1}$. In each case we will see that \# $\boldsymbol{A} \geqq \omega_{1}$ for $L_{\alpha, K} \cong \operatorname{Con} \boldsymbol{A}$ (and of course $\# \boldsymbol{A}=\omega_{1}$ is possible, by Grätzer-Schmidt).

For our first example we take $K=\omega_{1}^{*}$ ( $\omega_{1}$ upside down), and we apply Lemma 5 with $Z=0, \Psi=\alpha$ and $P=\{\{b, \alpha\}: \alpha \in K\}$. In this example $P$ is not a partition, and only a weak disjointness assumption (5) holds.

For our next example we take $K=K_{0} \cup\{p, c\}$, with $K_{0}=$ the lattice of all finite or co-countable subsets of $\omega_{1}$, and $p$ and $c$ two new points. The natural order of $K_{0}$ is extended to $K$ by letting $x \leqq c$ for all $x \in K$, and letting $x \leqq p$ if and only if $x$ is a finite subset of $\omega_{1}$. Let $\Psi=c, P=\left\{\{p, x\}: x\right.$ a coatom of $\left.K_{0}\right\}$ and $Z=$ the join in 
$L_{\omega_{1}, K}$ of all the atoms in $K_{0}$. Then one may easily check that $\Psi, P, Z$ satisfy the hypotheses of Lemma 5 (whenever $L_{\omega_{1}, K}$ is identified with Con $\boldsymbol{A}$ ). We could not have $Z$ any smaller and still satisfy (5), for if $Y<Z$, then $Y \geqq$ some atom $e \in K_{0}$, but clearly $e=\wedge T$ for a suitable set $T$ of coatoms.

For our third example we let $X=\left\{x_{\alpha}: \alpha<\omega_{1}\right\}$ and let $F L(X)$ be the free lattice generated by $X$, and then we take $K$ to be the lattice of dual ideals of $F L(X)$, ordered by reverse set inclusion. Let $\Psi \in K$ be the dual ideal of $F L(X)$ generated by the set $\left\{x_{0} \vee x_{\alpha}\right.$ : $\left.0<\alpha<\omega_{1}\right\}$. We will think of $F L(X)$ as a sublattice of $K$ (i.e., $K$ is a special kind of completion of $F L(X)$ ). Straightforward calculation shows that the hypotheses of Lemma 5 hold for $\left.\Psi, P=\left\{x_{0}, x_{\alpha}\right\}: 0<\alpha<\omega_{1}\right\}$ and $Z=$ the ideal of $K$ generated by $\left\{\wedge T: T \subseteq X,|T|=\omega_{1}\right\}$ (whenever $L_{\omega_{1}, K}$ is identified with Con $\boldsymbol{A}$ ). Note that in this example $\Psi$ and $Z$ are not comparable.

Of course, further examples could be obtained by embedding the ideals of $K$ into other lattices obeying (*), e.g., a subspace lattice. Incidentally, we are unable to decide whether $L_{\omega_{1}, \varnothing}$ or $L_{\omega_{1}, \omega_{1}}$ can be represented with $\aleph_{0}$ operations.

3. Further speculations. We first remark that obviously $(*)$ can be weakened without harm to

(**) For every compact $\Psi$ there exist compact $\Theta_{1}, \cdots, \Theta_{n}$ such that $\Theta_{1} \vee \cdots \vee \Theta_{n} \geqq \Psi$ and $\Theta_{1} \wedge \Psi=\cdots=\Theta_{n} \wedge \Psi=0$.

Notice also that if some principal dual ideal $D$ of $L$ requires $\kappa$ operations for a representation, then the same is true of $L$, since Con $A \cong L$ implies Con $A / \Theta \cong D$ for some $\Theta$. (And so one easily sees that every algebraic lattice $L$ is embeddable in one which requires $\kappa$ operations.) Thus in searching for a characterization of " $L$ is representable with $\leqq \kappa$ operations" we need to examine all principal dual ideals of $L$. Define $C(L)$ to be the set of compact elements of $L$ and then define

$$
\begin{aligned}
& \sigma(L)=\Sigma\{|\cup P|:(\exists \Psi, Z \in L) P, \Psi \text { and } Z \text { are as in Lemma 5, and } \\
& \text { there exists a compact element } \geqq \vee(\cup P) \vee Z\} \text {. } \\
& \tau(L)=\left\{\begin{array}{cl}
|C(L)| & \text { if } \exists X \subseteq C(L) \text { with }|X|<|C(L)| \text { and } \vee X=1 ; \\
0 & \text { otherwise . }
\end{array}\right. \\
& \rho(L)=\Sigma\{\sigma(D)+\tau(D): D \text { a principal dual ideal of } L \text { obeying }(* *)\} \text {. }
\end{aligned}
$$

Clearly all our previous results combine to show that $\# \boldsymbol{A}+\boldsymbol{\aleph}_{0} \geqq$ $\rho(\operatorname{Con} \boldsymbol{A})$. This last inequality summarizes all our available information on this topic. Thus, for example, we see that $\rho(L) \leqq \aleph_{0}$ is a necessary condition for congruence representability with $\aleph_{0}$ operations; but we would hardly conjecture that it is a sufficient condition. 


\section{Problems.}

(1) Is the inequality $\# \boldsymbol{A}+\boldsymbol{\aleph}_{0} \geqq \rho(\operatorname{Con} \boldsymbol{A})$ sharp, in the sense that every algebraic lattice is isomorphic to some $\operatorname{Con} \boldsymbol{A}$ with $\# \boldsymbol{A}+$ $\boldsymbol{\aleph}_{0}=\rho(\operatorname{Con} \boldsymbol{A})+\boldsymbol{\aleph}_{0}$ ? (See $\S 3$ just above.)

(2) If no principal dual ideal of $L$ obeys $(* *)$ of $\S 3$, is $L$ representable with $\leqq \boldsymbol{W}_{0}$ operations? (See § 3.)

(3) Is every distributive algebraic lattice $L$ representable with $\aleph_{0}$ operations? ... one operation? ... as Con (some lattice)? This last property holds for $L$ finite [1, p. 83] [4, p. 95]. (See also [2], [5], [15]-[17] for further information.) A groupoid representation exists if $L$ is the ideal lattice of a distributive lattice (see Part II of this paper).

Notice that if 1 is true, then so is 2 , and if 2 is true, then so is the first part of 3 . We would be surprised if 1 is true.

(4) If the set of compact elements of $L$ is countable, then is $L$ representable with $<\boldsymbol{\aleph}_{0}$ operations?

(5) If below each compact element of $L$ there are $\leqq \aleph_{0}$ compact elements, then is $L$ representable with $\boldsymbol{\aleph}_{0}$ operations? . . < $\boldsymbol{\aleph}_{0}$ operations? ... one operation? (W. Hanf showed [7] (see also [8, p. 106]) that the corresponding assertions hold for representations as subalgebra lattices.)

(6) If $\boldsymbol{A}$ has countable similarity type, must there exist $\boldsymbol{B}$ of finite similarity type with $\operatorname{Con} \boldsymbol{B} \cong \operatorname{Con} \boldsymbol{A}$ ?

(7) If $\boldsymbol{A}$ has finite similarity type, must there exist a groupoid $\boldsymbol{B}$ with Con $\boldsymbol{B} \cong \operatorname{Con} \boldsymbol{A}$ ? (Lampe conjectures yes.) Note that Hanf [7] proved the corresponding assertion for subalgebra lattices, and McKenzie has proved a slightly weaker assertion for Con: there exists $\boldsymbol{B}$ of type $(2,1)$ with $\operatorname{Con} \boldsymbol{B} \cong \operatorname{Con} \boldsymbol{A}$ (see Lampe's survey paper in the Proceedings of the 1977 Esztergom Colloquium).

(8) Characterize the class of lattices isomorphic to congruence lattices of groupoids. $\ldots$ of algebras with $<\kappa$ operations.

\section{REFERENCES}

1. P. Crawley and R. P. Dilworth, Algebraic Theory of Lattices, Prentice-Hall, Englewood Cliffs, N. J., 1973.

2. R. Freese, Congruence lattices of finitely generated modular lattices, Proc. Lattice Theory Conf., U1m 1975, G. Kalmbach, Editor, 62-70.

3. G. Grätzer, Universal Algebra, D. Van Nostrand Co., Inc., Princeton, N. J., 1968.

4. - Lattice Theory, First concepts and distributive lattices, H. M. Freeman, San Francisco, 1971.

5. G. Grätzer and E. T. Schmidt, On congruences of lattices, Acta Math. Acad. Sci. Hung., 13 (1962), 179-185.

6. Characterizations of congruence lattices of abstract algebras, Acta Sci. Math. (Szeged), 24 (1963), 34-59.

7. W. Hanf, Representations of lattices of subalgebras (Preliminary report), Bull. Amer. Math. Soc., 62 (1956), 402. 
8. B. Jónsson, Topics in Universal Algebra, Lecture Notes in Mathematics vol. 250, Springer-Verlag, Berlin, 1972.

9. - Varieties of algebras and their congruence varieties, Pros. Int. Congress Math., Vancouver 1974, 315-320.

10. W. A. Lampe, The independence of certain related structures of a universal algebra I-IV, Alg. Univ., 2 (1972), 99-112, 270-283, 286-295, 296-302.

11. - Notes on the congruence lattices of algebras of finite type, (1975 manuscript).

12. - A note on algebras of fixed similarity type, Abstract 77T-A120, Notices Amer. Math. Soc., 24 (1977), A-371.

13. — Review of B. Jónsson [9], MR 55 (1978). \#241.

14. P. Pudlák, A new proof of the congruence lattice representation theorem, Algebra Universalis, 6 (1976), 269-275.

15. E. T. Schmidt, Kongruenzrelationen algebraischer Strulturen, Mathematische Forschungsberichte, VEB Deutscher Verlag Berlin, 1969, MR 47 \#136.

16. - Every finite distributive lattic is the congruence lattice of a modular lattice, Algebra Universalis, 4 (1974), 49-57.

17. Congruence lattices of complemented modular lattices, Proceedings of the colloquium on universal algebra, Esztergom 1977, (to appear).

18. W. Taylor, Congruence representation of modular lattices, Abstract 77T-A173, Notices Amer. Math. Soc., 24 (1977), A-420.

Received June 7, 1978

UNIVERSITY OF HAWAII AT MANOA

HONOLULU, HI 96822

AND

UNIVERSITY OF COLORADO

BOULDER, CO 80309 


\section{PACIFIC JOURNAL OF MATHEMATICS}

\section{EDITORS}

DoNALD BABBITT (Managing Editor)

University of California

Los Angeles, California 90024

HUGo Rossi

University of Utah

Salt Lake City, UT 84112

C. C. MOORE and ANDREW OGG

University of California

Berkeley, CA 94720
J. DUGUNDJI

Department of Mathematics University of Southern Californı Los Angeles, California 90007

R. Finn and J. Milgram Stanford University Stanford, California 94305

\section{ASSOCIATE EDITORS}

E. F. BECKENBACH

B. H. Neumann
F. WOLF

K. YOSHIDA

\section{SUPPORTING INSTITUTIONS}

UNIVERSITY OF BRITISH COLUMBIA CALIFORNIA INSTITUTE OF TECHNOLOGY UNIVERSITY OF CALIFORNIA MONTANA STATE UNIVERSITY UNIVERSITY OF NEVADA, RENO NEW MEXICO STATE UNIVERSITY OREGON STATE UNIVERSITY UNIVERSITY OF OREGON
UNIVERSITY OF SOUTHERN CALIFORNIA STANFORD UNIVERSITY UNIVERSITY OF HAWAII UNIVERSITY OF TOKYO UNIVERSITY OF UTAH WASHINGTON STATE UNIVERSITY UNIVERSITY OF WASHINGTON 


\section{Pacific Journal of Mathematics}

Vol. 82, No. $1 \quad$ January, 1979

Werner Bäni, Subspaces of positive definite inner product spaces of countable dimension ...................................... 1

Marilyn Breen, The dimension of the kernel of a planar set..............

Kenneth Alfred Byrd, Right self-injective rings whose essential right ideals

are two-sided

Patrick Cousot and Radhia Cousot, Constructive versions of Tarski's fixed

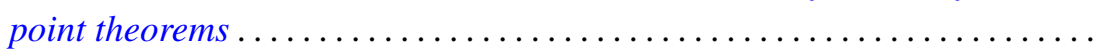

Ralph S. Freese, William A. Lampe and Walter Fuller Taylor, Congruence lattices of algebras of fixed similarity type. $I \ldots \ldots \ldots \ldots \ldots \ldots \ldots$

Cameron Gordon and Richard A. Litherland, On a theorem of Murasugi .....

Mauricio A. Gutiérrez, Concordance and homotopy. I. Fundamental

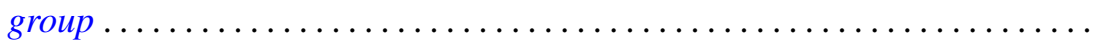

Richard I. Hartley, Metabelian representations of knot groups .............

Ted Hurley, Intersections of terms of polycentral series of free groups and free

Lie algebras ........................................

Roy Andrew Johnson, Some relationships between measures ............ 117

Oldřich Kowalski, On unitary automorphisms of solvable Lie algebras .......

Kee Yuen Lam, $K O$-equivalences and existence of nonsingular bilinear

maps...................................................

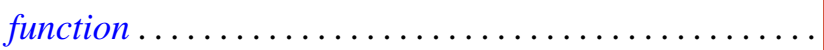

Robert A. Messer and Alden H. Wright, Embedding open 3-manifolds in compact 3-manifolds ............................

Gerald Ira Myerson, A combinatorial problem in finite fields. I . .

James Nelson, Jr. and Mohan S. Putcha, Word equations in a band of paths.

Baburao Govindrao Pachpatte and S. M. Singare, Discrete generalized Gronwall inequalities in three independent variables . .

William Lindall Paschke and Norberto Salinas, $C^{*}$-algebras associated with free products of groups ........................

Bruce Reznick, Banach spaces with polynomial norms ....

David Rusin, What is the probability that two elements of a finite group

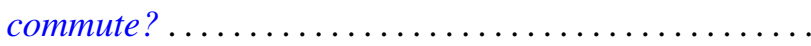

M. Shafii-Mousavi and Zbigniew Zielezny, On hypoelliptic differential operators of constant strength ...

Joseph Gail Stampfli, On selfadjoint derivation ranges .... . . .

Robert Charles Thompson, The case of equality in the matrix-valued triangle

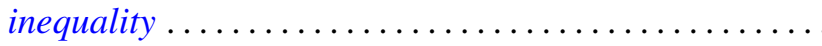

Marie Angela Vitulli, The obstruction of the formal moduli space in the negatively graded case. 